\title{
HSPA I2B Secreted by Tumor-Associated Endothelial Cells Might Induce M2 Polarization of Macrophages via Activating PI3K/Akt/mTOR Signaling
}

This article was published in the following Dove Press journal: OncoTargets and Therapy

\section{Jingjie Zhou* \\ Aiping Zhang* \\ Liang Fan}

Department of OtorhinolaryngologyHead and Neck Surgery, Jingmen No. I People's Hospital, Jingmen, Hubei 448000, People's Republic of China

*These authors contributed equally to this work
Correspondence: Liang Fan $\mathrm{Tel} / \mathrm{Fax}+867242305984$

Email 10384757@qq.com
Purpose: The intratumoral microenvironment of head and neck squamous cell carcinoma (HNSC) is highly immunosuppressive. In this study, we explored the potential functional role of HSPA12B secreted by tumor-associated endothelial cells (TECs) in M2 polarization of macrophages.

Materials and Methods: Bulk-seq data from TCGA-HNSC and single-cell RNA-seq data from GSE103322 (with over 5000 cells from 18 primary HNSC cases) were used for bioinformatic analysis. RAW264.7 cell line was used for in vitro studies.

Results: TECs in HNSC had significantly higher expression and secretion of HSPA12B, compared to normal human umbilical vein endothelial cells (HUVECs). Exogenous HSPA12B treatment increased the expression of M2 macrophage marker CD163 and CD206 on RAW264.7 cells in a dose-dependent manner but had no significant influence on CD86, an M1 macrophage marker. OLR1, a known receptor of HSP70 proteins, was specifically expressed in tumor-associated macrophages (TAMs) in HNSC. OLR1 knockdown significantly impaired HSPA12B uptake by RAW264.7 cells and weakened HSPA12Binduced CD163 and CD206 upregulation. HSPA12B treatment increased the expression of p-PI3K, p-Akt and p-mTOR in a dose-dependent manner in RAW264.7 cells. OLR1 inhibition and LY294002 treatment significantly weakened the effects HSPA12B on activating the $\mathrm{PI} 3 \mathrm{~K} / \mathrm{Akt} / \mathrm{mTOR}$ signaling and M2 marker expression.

Conclusion: Based on these findings, we speculated that aberrantly expressed and secreted HSPA12B by TECs could be taken by macrophages partly via OLR1, leading to subsequent activation of the PI3K/Akt/mTOR signaling pathway and elevated expression of M2 markers. This mechanism shows a novel cross-talk between TECs and TAMs, which contributes to the intratumoral immunosuppressive microenvironment.

Keywords: HSPA12B, OLR1, head and neck squamous cell carcinoma, macrophage polarization

\section{Introduction}

Head and neck squamous cell carcinoma (HNSC) is a group of heterogeneous tumors that originated from different anatomic sites, including the tonsil, oral cavity, hypopharynx, oropharynx, nasopharynx or larynx. The microenvironment of HNSC is highly immunosuppressive, with significantly increased myeloid-derived suppressor cells (MDSCs), regulatory $\mathrm{T}$ cells (Tregs), as well as dysfunctional effector $\mathrm{CD} 8^{+}$ T cells. ${ }^{1}$ Currently, immune-checkpoint inhibitors (ICI) targeting CTLA4, PD-1, and PD-L1 have shown great survival benefits for multiple types of cancers, including 
HNSC. ${ }^{2,3}$ However, the overall response rate is only around $13-18 \%{ }^{3}$ Several mechanisms have been proposed to explain the resistance to anti-PD-1/PD-L1 therapy, including low intratumoral immune cell infiltration, the presence of immune inhibitory receptors, and the induced immunosuppressive tumor microenvironment. ${ }^{4,5}$

In tumor microenvironment, tumor-infiltrating macrophages (TIMs) can be generally divided into an immunostimulatory M1 subset that exerts proinflammatory and antitumoral effects, and an immunoregulatory M2 subset that secretes immunosuppressive cytokines (such as IL-10 and TGF- $\beta$ ) and shows tumor-promoting function. ${ }^{6}$ In many types of tumors, the M2 subset accounts for the dominant proportion of TIMs and inhibits the differentiation of M1 macrophage. ${ }^{7}$ In HNSC, M2 macrophages promote cellular dedifferentiation, angiogenesis and subsequent cancer progression, relapse and poor prognosis. ${ }^{6,8}$ Therefore, some strategies targeting M2 macrophages have been under testing in clinical settings, such as inhibiting TIM differentiation, ${ }^{9}$ blocking TIM activation ${ }^{10}$ and reprogramming TIMs to M1. ${ }^{11}$

The 70-kDa heat shock proteins (Hsp70s) are a group of ubiquitous molecular chaperones involved in a wide range of cellular protein folding and remodeling processes, thereby modulating cellular responses to stress conditions. ${ }^{12}$ Unlike most of other heat shock proteins with ubiquitous expression, HSPA12B is specifically expressed by endothelial cells. ${ }^{13}$ Functionally, it protects endothelial cell integrity under multiple stress conditions, including lipopolysaccharide (LPS)-induced sepsis, ${ }^{14}$ acute myocardial ischemia/ reperfusion-induced injury, ${ }^{15}$ and ischemic stroke. ${ }^{16}$ These findings collectively suggest that HSPA12B might have a critical role in suppressing inflammation responses. However, its potential involvement in tumor immune microenvironment has not been investigated.

In this study, we explored the expression profiles Hsp70s in different HNSC cell groups using one previous single-cell (sc)-RNA-seq data and further studied the effect of HSPA12B secreted by tumor-associated endothelial cells (TECs) on macrophage polarization.

\section{Materials and Methods}

\section{Sc-RNA-seq Data Retrieved from} GSEI03322

The RNA expression profile of HSP70 family members, OLR1, CD163 and CD206 at the single-cell level was examined using transcriptomic data from GSE103322, which included over 5000 cells from 18 primary HNSC cases in GSE103322. ${ }^{17}$ Briefly, this dataset included over 2000 tumor cells, 1440 fibroblasts, 260 endothelial cells, and 98 macrophages. Normalized data were analyzed using the UCSC Xena browser (https://xenabrowser.net/). OLR1 expression (Transcript per million, TPM) in each cell group was compared. TPM $\geq 1$ was set as the threshold of gene expression.

\section{Data Retrieved from TCGA-Head and Neck Squamous Cell Carcinoma (HNSC)} The gene-expression data (by bulk-seq) in TCGA-HNSC dataset was acquired using the UCSC Xena browser. Gene expression was presented as $\log 2(\mathrm{RESM}+1)$.

\section{Isolation of Primary Tumor-Associated Endothelial Cells (PTECs)}

PTECs were isolated from the tumor tissues from a patient with primary laryngeal SCC (LSCC), who underwent surgical resection Jingmen No. 1 People's Hospital, Hubei, China. Informed consent and ethical approval were acquired from the Ethics Committee of Jingmen No. 1 People's Hospital before this study. PTEC isolation was performed by using Dynabeads CD31 and DynaMag-5 magnet (Invitrogen Life Technologies, CARLSBAD, CA, USA), according to the protocol recommended by the manufacturer. Briefly, $1 \mathrm{~mL}$ single-cell suspensions containing $1 \times 10^{8}$ cells were prepared from tumor tissue as a starting sample. Then, $25 \mathrm{uL}$ pre-washed and resuspended Dynabeads were added and were incubated $30 \mathrm{~min}$ on ice, with gentle tilting and rotation. Then, the tube was placed in a magnet for $2 \mathrm{~min}$. The supernatant was removed and the tube was washed twice. Then, beadbound $\mathrm{CD} 31+$ endothelial cells were mashed, resuspended and cultured in cell medium.

\section{Cell Culture and Treatment}

Since the cells in GSE103322 are all from tongue squamous cell carcinoma (TSCC), we used two TSCC cell lines (SCC9 and SCC25), which were purchased from the American Type Culture Collection (Manassas, VA, USA) as representative tumor cell model. The RAW264.7 cell line and primary human umbilical vein endothelial cells (HUVECs) were obtained from the Cell Bank of the Chinese Academy of Sciences (Shanghai, China). RAW 264.7 cells are a macrophage-like cell line that has been widely used to study macrophage polarization. ${ }^{18,19}$ RAW264.7 cells were cultured in high-glucose Dulbecco's 
Modification of Eagle's Medium (DMEM) (HyClone, Logan, UT, USA) with 10\% fetal bovine serum (FBS) and $100 \mathrm{U} / \mathrm{mL}$ penicillin, and $100 \mu \mathrm{g} / \mathrm{mL}$ streptomycin (Clark Bioscience, Seabrook, MD, USA). PTECs and HUVECs were cultured in endothelial cell medium (ECM, Sciencell, San Diego, CA, USA) supplemented with 5\% FBS, 1\% endothelial cell growth supplements (ECGS), $100 \mathrm{U} / \mathrm{mL}$ penicillin, and $100 \mu \mathrm{g} / \mathrm{mL}$ streptomycin. Primary cells between passages 3 and 5 were used in experiments. SCC9 and SCC25 cells were cultured in DMEM/F12 containing 10\% fetal bovine serum (FBS) and $400 \mathrm{ng} / \mathrm{mL}$ hydrocortisone. All cells were cultured at $37^{\circ} \mathrm{C}$ under $5 \%$ $\mathrm{CO}_{2}$ in a humidified incubator.

Lentiviral plasmids carrying OLR1 shRNA (shOLR1\#1, with siRNA sequence, 5'-ACAGCTATATTCA TCAGGCAA-3' and sh-OLR1\#2, with siRNA sequence, 5'-AGAAGCCTAAAGGTCTGCATT-3') and control plasmid (shNC) carrying scramble sequence were constructed by HanBio Technology (Shanghai, China). Lentiviral particles were amplified by co-transfecting with the lentivirus packaging plasmids (pSPAX2 and pMD2G, HanBio Technology) in 293 T cells according to the manufacturer's instructions. $48 \mathrm{~h}$ after transfection, the supernatant was harvested, calibrated and subjected to viral titer estimation. RAW264.7 cells were infected with the lentiviral vectors at a multiplicity of infection (MOI) of 10. Recombinant HSPA12B protein was purchased from Zeye Biotechnology (Shanghai, China).

\section{Labeling HSPA I2B with FITC}

The binding of FITC and recombinant HSPA12B was conducted using the $\mathrm{HOOK}^{\mathrm{TM}}$ Dye Labeling Kit (FITC) (\#786-141, G-Biosciences, St Louis, MO, USA), according to the manufacturer's instructions.

\section{Immunofluorescence (IF) Staining}

PTECs and RAW264.7 cells grown on sterile coverslips were fixed with $4 \%$ paraformaldehyde for 15 minutes at room temperature and permeabilized with $0.5 \%$ Triton X-100 for 10 minutes. Blocking was performed using 3\% BSA for 30 minutes. Then, cells were incubated with the primary antibodies against CD31 (PECAM) (1:1000, 11-0311-82, ThermoFisher, Waltham, MA, USA), HSPA12B (1:500, HPA015639, Sigma-Aldrich, St. Louis, MO, USA) or OLR1 (1:500, PA5-102,452, ThermoFisher) for overnight at $4{ }^{\circ} \mathrm{C}$. After that, the coverslips were rinsed and incubated with the secondary antibody conjugated to Alexa Fluor ${ }^{\circledR} 488$ conjugated or Alexa Fluor ${ }^{\circledR} 647$ conjugated secondary antibodies at 1:1000 dilution for $1 \mathrm{~h}$ at room temperature in the dark. DNA was counterstained with Hochest 33,342. Coverslips were then mounted with Fluoromount-G. Fluorescence images were acquired with a confocal microscope (Zeiss LSM710, Heidenheim, Germany).

\section{Quantitative Real-Time Polymerase Chain Reaction (qRT-PCR) Analysis}

qRT-PCR was used to examine mRNA expression. Total was isolated with RNAiso Plus (Takara, Tokyo, Japan). Then, cDNA was synthesized using PrimeScript ${ }^{\mathrm{TM}}$ RT Master Mix (Perfect Real Time; Takara) following the manufacturer's instructions. qRT-PCR was performed on 5-folddiluted cDNA samples in double-distilled water using SYBR $^{\circledR}$ Premix Ex Taq ${ }^{\mathrm{TM}}$ II (Tli RNaseH Plus, Takara). Each reaction contained $5 \mu \mathrm{L}$ of SYBR mix, $2 \mu \mathrm{L}$ of cDNA, $2.2 \mu \mathrm{L}$ of $\mathrm{ddH}_{2} \mathrm{O}, 0.4 \mu \mathrm{L}$ of forward primer and 0.4 $\mu \mathrm{L}$ of reverse primer. Primers for human HSPA12B were: forward, 5'-GCTGTGAAAGTCCTGTAATA-3' and reverse, 5'-AAAGTATAGCCAATGTCTGG-3'. Glyceraldehyde 3phosphate dehydrogenase (GAPDH) was used to normalize gene expression. Gene expression fold change was calculated by the $2^{-\triangle \Delta C T}$ method.

\section{Western Blot Analysis}

Protein extraction Kit (Beyotime, Shanghai, China) was used to extract total proteins. Then, $25 \mu \mathrm{g}$ proteins were separated by SDS-PAGE gel electrophoresis and transferred onto a polyvinylidene fluoride (PVDF) membrane. After blocking with 5\% skimmed milk, the membranes were probed with primary antibodies against HSPA12B (1:1000, HPA015639, Sigma-Aldrich), PI3K (p85 $\alpha)$ (1:1000, ab191606 Abcam, Cambridge, UK), p-PI3K (p85a, phospho Y607) (1:1000, ab182651, Abcam), AKT (1:1000, \#4685, Cell signaling, Danvers, MA, USA), p-AKT (Ser473, 1:1000, \#9271, Cell signaling), mTOR (1:1000, \#2983, Cell signaling), p-mTOR (Ser2448, 1:1000, \#5536, Cell signaling), and $\beta$-actin $(1: 1000$, AM4302, ThermoFisher). The species-matched HRPconjugated secondary antibodies were used (1:10,000). Protein bands were detected by the Odyssey imaging system (LI-COR, Lincoln, NE, USA).

\section{ELISA Assay of HSPA I2B Secretion}

HSPA12B concentration in the culture medium of PTECs, HUVECs, SCC9 and SCC25 cells was analyzed by ELISA assay (CSB-EL010818HU, Houston, TX, USA) according 
to manufacturer's instruction. Cells were plated at a density of $2 \times 10^{6}$ cells per $\mathrm{mL}$ in 24 -well plates with serum-free medium. $48 \mathrm{~h}$ after seeding, supernatants were harvested for ELISA assay.

\section{Flow Cytometric Assessment of CD86, CDI63, and CD206 Expression}

RAW264.7 cells were collected after indicated treatments. The cell surfaces were blocked with $15 \%$ sheep serum at $4{ }^{\circ} \mathrm{C}$ for $15 \mathrm{~min}$ and were then washed twice with phosphate buffer solution (PBS, pH7.2). Then, cells were incubated with monoclonal antibodies against CD86, CD163, CD206, and the corresponding fluorescent markers for $30 \mathrm{~min}$ at $4^{\circ} \mathrm{C}$. Then, the cells were washed twice, resuspended in PBS and were subjected to flow cytometry with a FACSVerse (Becton Dickinson, Franklin Lakes, NJ, USA).

\section{Statistical Analysis}

Data were reported in the form of means \pm standard deviation (SD). Statistical analysis was performed with the use of GraphPad Prism 8.04 (GraphPad Inc., La
Jolla, CA, USA) and SPSS 25.0 software package (SPSS Inc., Chicago, IL, USA). Group difference was assessed using the unpaired $t$-test. One-way ANOVA with post hoc Tukey's multiple comparisons test was performed for multiple group comparisons. Correlation coefficients were determined using Pearson's test. $p$-value of $<0.05$ was considered statistically significant.

\section{Results \\ PTECs Had Significantly Elevated HSPA I2B Expression and Secretion Compared to HUVECs}

Using sc-RNA-seq data from GSE103322, we examined the expression profile of 14 HSP70 genes in different HNSC tumor subgroups (Figure 1A). Results confirmed endothelial cell specific HSPA12B expression (Figure 1A, gray arrow). Then, we isolated PTECs from resected HNSC tumors from a patient with LSCC. IF staining confirmed the high level of CD31 (PECAM1), as well as HSPA12B expression in the PTECs (Figure 1B). QRT-PCR results indicated that HSPA12B mRNA expression was significantly higher in
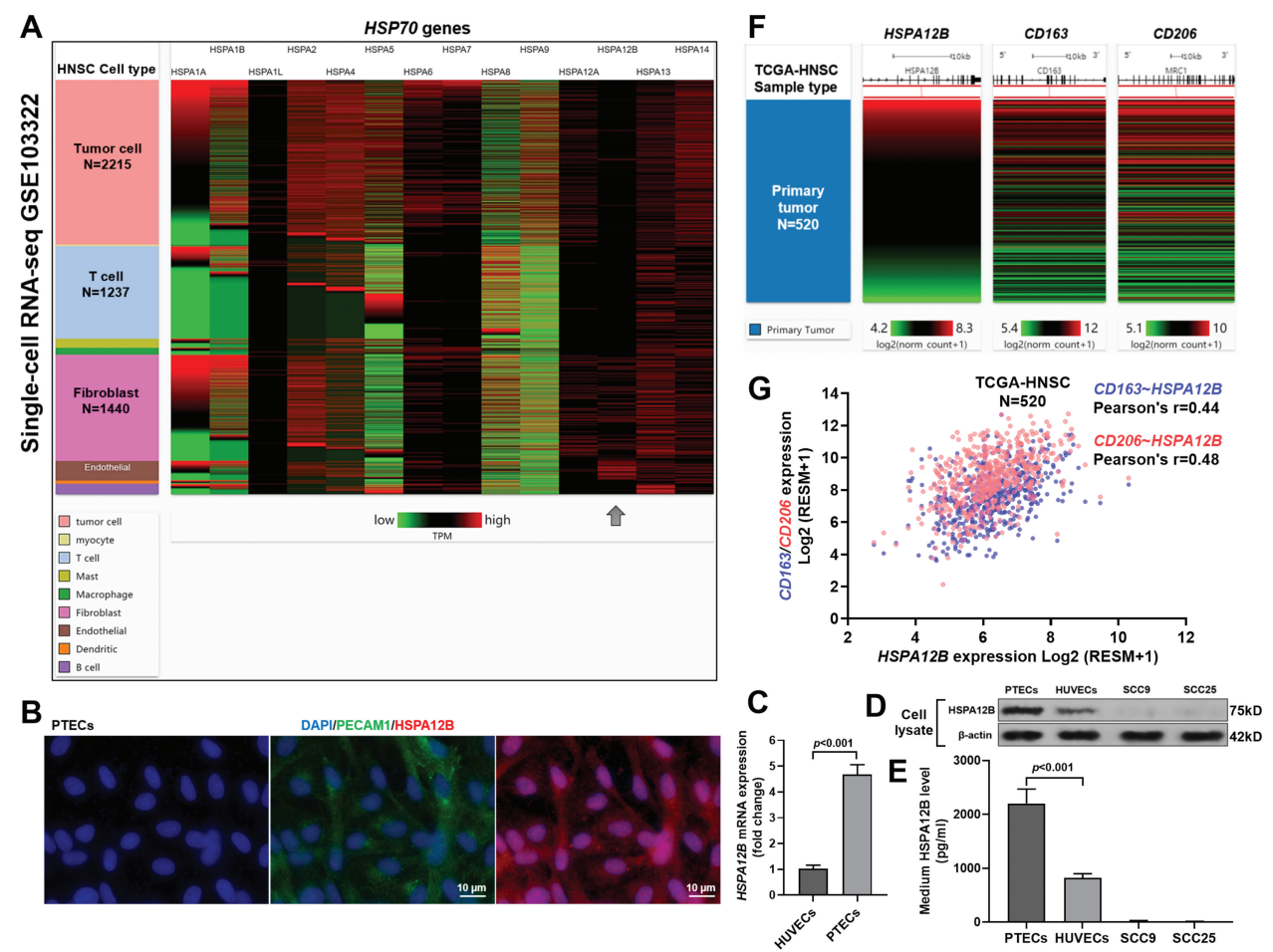

Figure I PTECs had significantly elevated HSPAI2B expression and secretion compared to HUVECs. (A) A heatmap showing the expression of HSP70 gene expression in different types of cells within HNSC tumors, using single-cell RNA-seq data from GSEI03322. The number of cells in the major cell groups was as indicated. HSPAI2B (dark gray arrow) showed restricted expression in endothelial cells. (B) IF staining of the nucleus (blue), PECAM (CD3I, green) HSPAI2B (red) in PTECs isolated from primary HNSC tumor tissues. (C) QRT-PCR analysis of HSPA I2B mRNA expression in PTECs and HUVECs. (D and E) Western blot (D) and ELISA (E) assay of HSPAI2B protein expression in cell lysate (D) and culture medium (E) of endothelial cells (PTECs and HUVECs) and TSCC cells (SCC9 and SCC25). (F and G) A heatmap (F) and a plot chart (G) showing the correlation between HSPA I2B expression and two M2 macrophage markers (CDI63 and CD206) at the RNA level cases in primary tumor cases (N=520) in TCGA-HNSC. 
PTECs than in HUVEC cells (Figure 1C). Western blot and ELISA assay confirmed that PTECs had significantly higher intracellular and secreted HSPA12B, compared to HUVEC cells (Figure 1D and E). Using data from primary tumor cases $(\mathrm{N}=520)$ in TCGA-HNSC, we further assessed the correlation between HSP70 family members and two M2 macrophage markers, CD163 and MRC1 (also known as CD206) (Supplementary Figure 1A and B). Analysis showed that only HSPA12B expression had a moderate correlation with the two M2 macrophage markers (Pearson's $r>0.4$, Supplementary Figure 1B and Figure 1F and G). Therefore, this gene was selected for further analysis.

\section{Exogenous HSPAI2B Treatment \\ Stimulated the Expression of $M 2$} Macrophages-Associated Surface Markers

Using RAW264.7 cells as an in-vitro macrophage model, we assessed the influence of HSPA12B treatment on macrophage polarization. Results showed that HSPA12B treatment increased the expression of M2 macrophage marker CD163 and CD206 on RAW264.7 cells in a dosedependent manner (Figure 2C-F). In contrast, we did not detect any significant increase of CD86 expression by exogenous HSPA12B treatment (Figure 2A and B).

\section{The Potential M2 Activating Effects of HSPA I2B Were Mediated by OLR I}

Previous studies reported that OLR1 is a receptor of Hsp70 proteins, ${ }^{20}$ which has a specific expression in macrophages. ${ }^{21,22}$ Using sc-RNA-seq data from GSE103322, we found that a large proportion of tumorassociated macrophages (TAMs) (TPM $\geq 1,63.3 \%)$ had OLR1 expression (Figure 3A). Correlation analysis showed a moderate positive correlation between OLR1 and CD206 (Pearson's r $=0.47, p<0.001$, Figure 3D) and a weak positive correlation between OLR1 and CD163

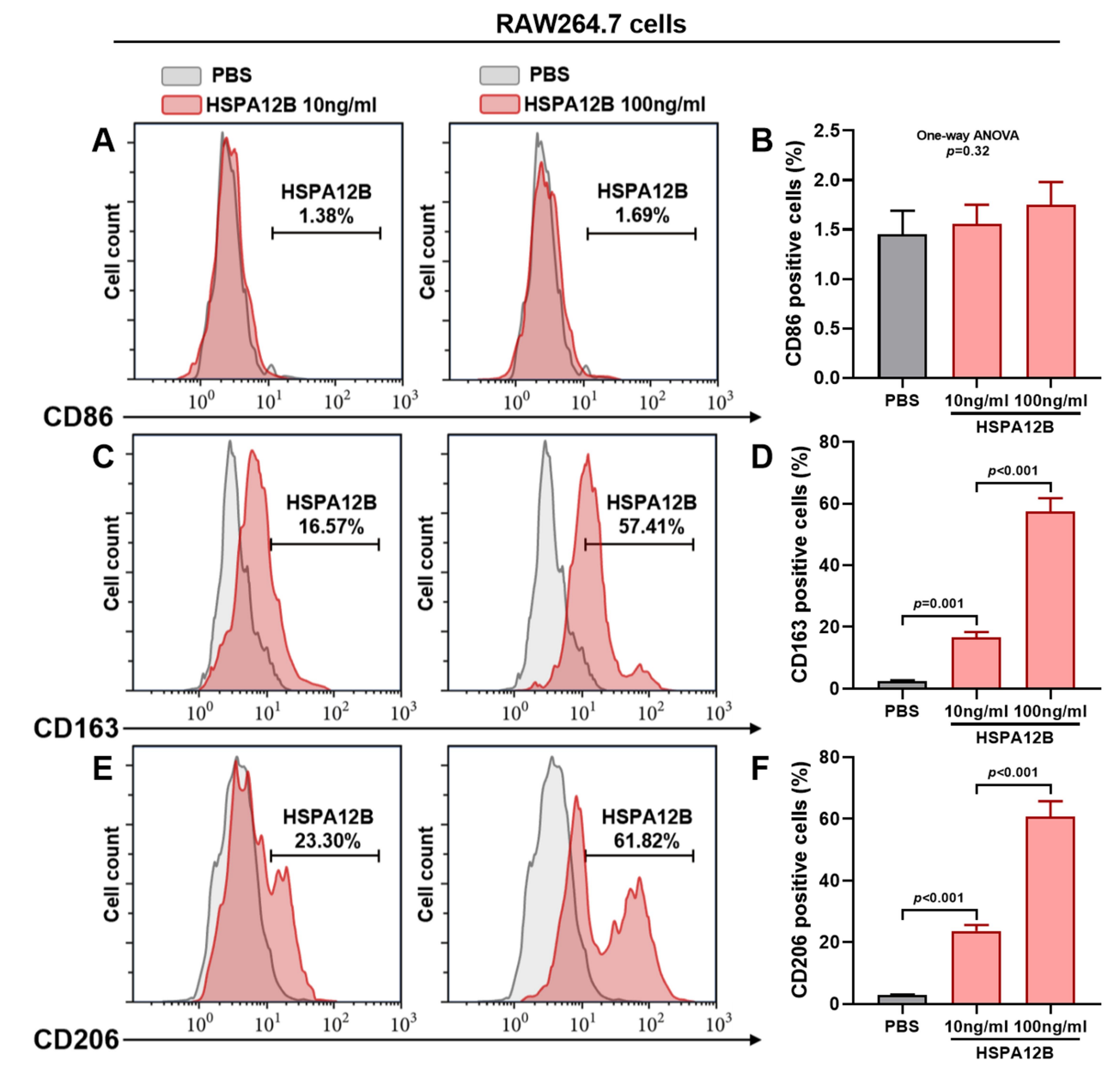

Figure 2 Exogenous HSPAI2B treatment stimulated the expression of M2 macrophages-associated surface markers Representative images (A, C and E) and statistical analysis of cell surface markers for MI macrophages (CD86) (A and B) and M2 macrophages (CDI63 and CD206) (C-F) in RAW264.7 cells 48h after treatment with I0ng/ $\mathrm{mL}$ or $100 \mathrm{ng} / \mathrm{mL}$ HSPAI2B. RAW264.7 cells with PBS added were served as control. 

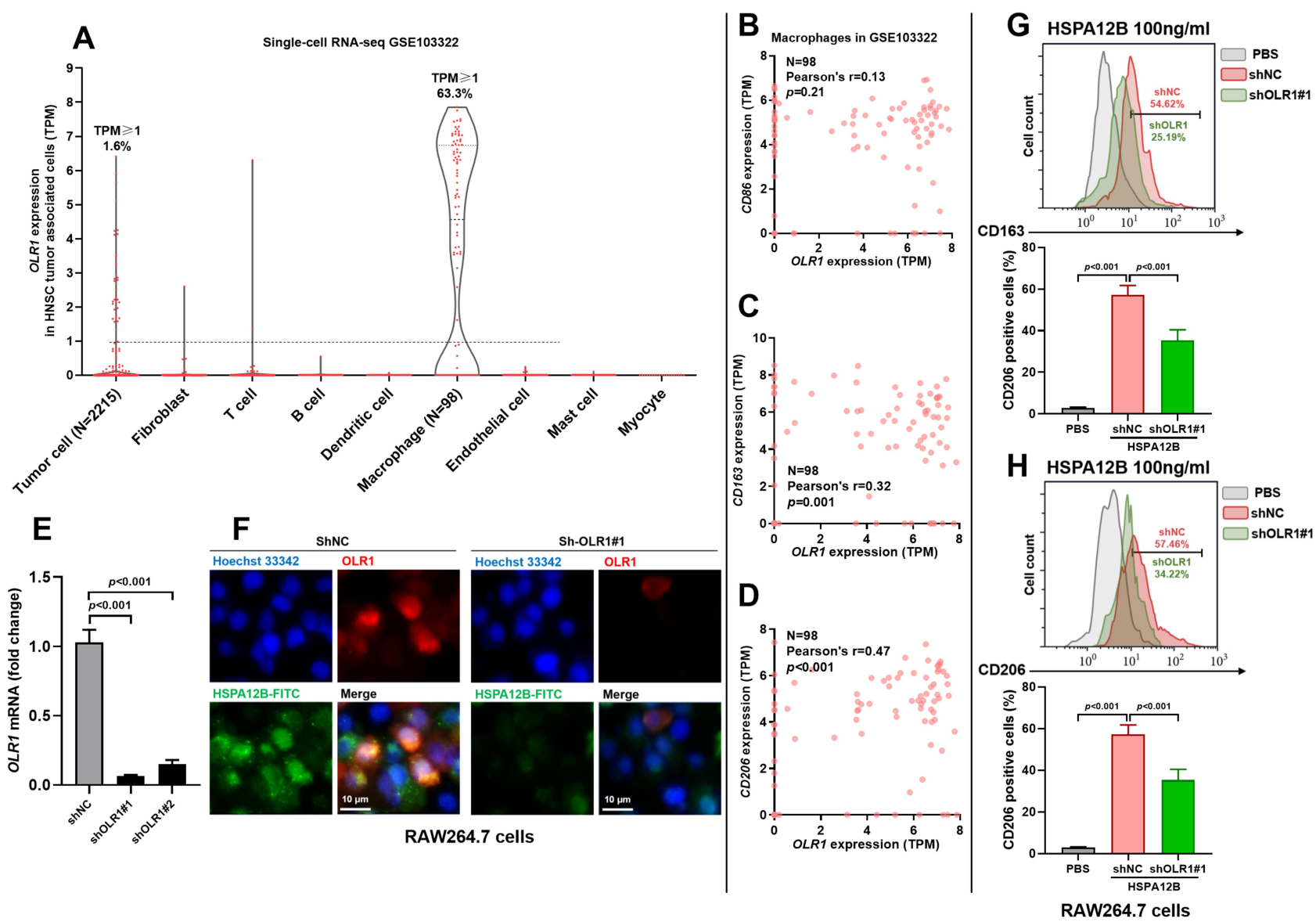

Figure 3 The potential M2 activating effects of HSPAI2B were mediated by OLRI. (A) A violin plot chart showing the expression of OLRI in different types of cells within HNSC tumors, using sc-RNA-seq data from GSEI03322. (B and D) Correlation analysis between OLRI expression and CD86 (B), CDI63 (C) and CD206 (D) in 98 tumor-associated macrophages in GSEI03322. (E) QRT-PCR analysis of OLRI expression in RAW264.7 cells with or without OLRI knockdown. (F) 48h after lentiviral infection, RAW264.7 cells were pretreated for Ih with $200 \mathrm{ng} / \mathrm{mL}$ HSPAI2B-FITC before fixation and labeling with the OLRI monoclonal antibody. Immunofluorescence staining was conducted to examine the expression of OLRI (red) and HSPAI2-FITC uptake (green). (G and H) Representative images (up) and Statistical analysis (down) of markers for M2 macrophages, CDI63 (G) and CD206 (H) in RAW264.7 cells with or without OLRI knockdown $48 \mathrm{~h}$ after treatment with $100 \mathrm{ng} / \mathrm{mL}$ HSPAI2B.

(Pearson's $\mathrm{r}=0.32, p=0.001$, Figure 3C). However, no significant correlation was observed between OLR1 and CD86 (Pearson's $\mathrm{r}=0.13, p=0.21$, Figure 3B). To valid the functional role of OLR1 in macrophage polarization, RAW264.7 cells were subjected to OLR1 knockdown (Figure 3E). OLR1 knockdown significantly reduced the uptake of exogenous HSPA12B by RAW264.7 cells (Figure 3F), as well as HSPA12B induced CD163 and CD206 upregulation (Figure 3G and $\mathrm{H}$ ).

\section{HSPA I2B Activates the PI3K/Akt/mTOR} Signaling in Macrophages

Previous studies found that HSPA12B is a potential activator of $\mathrm{PI} 3 \mathrm{~K} / \mathrm{Akt} / \mathrm{mTOR}$ signaling in endothelial cells and in some cancer cells. ${ }^{23,24}$ Actually, activation of the $\mathrm{PI} 3 \mathrm{~K} / \mathrm{Akt} / \mathrm{mTOR}$ signaling is critical for M2 polarization of macrophages. ${ }^{25}$ Therefore, we further assessed whether exogenous HSPA12B could activate the PI3K/Akt/mTOR signaling in macrophages. By performing Western blot assay, we showed that HSPA12B treatment increased the expression of p-PI3K, p-Akt and p-mTOR in a dosedependent manner in RAW264.7 cells (Figure 4A). In RAW264.7 cells with OLR1 inhibition, HSPA12B treatment had significantly weakened effects on activating the $\mathrm{PI} 3 \mathrm{~K} / \mathrm{Akt} / \mathrm{mTOR}$ signaling and M2 marker expression (Figure 4B). Western blot and flow cytometry data confirmed that LY294002 (a typical selective inhibitor of PI3K) treatment also impaired HSPA12B induced M2 macrophage marker expression (Figure 4B-D). Based on these findings, we infer that HSPA12B secreted by TECs could be transferred to macrophages partly via OLR1, leading to subsequent activation of the PI3K/Akt/mTOR signaling pathway and M2 polarization (Figure 4E). 

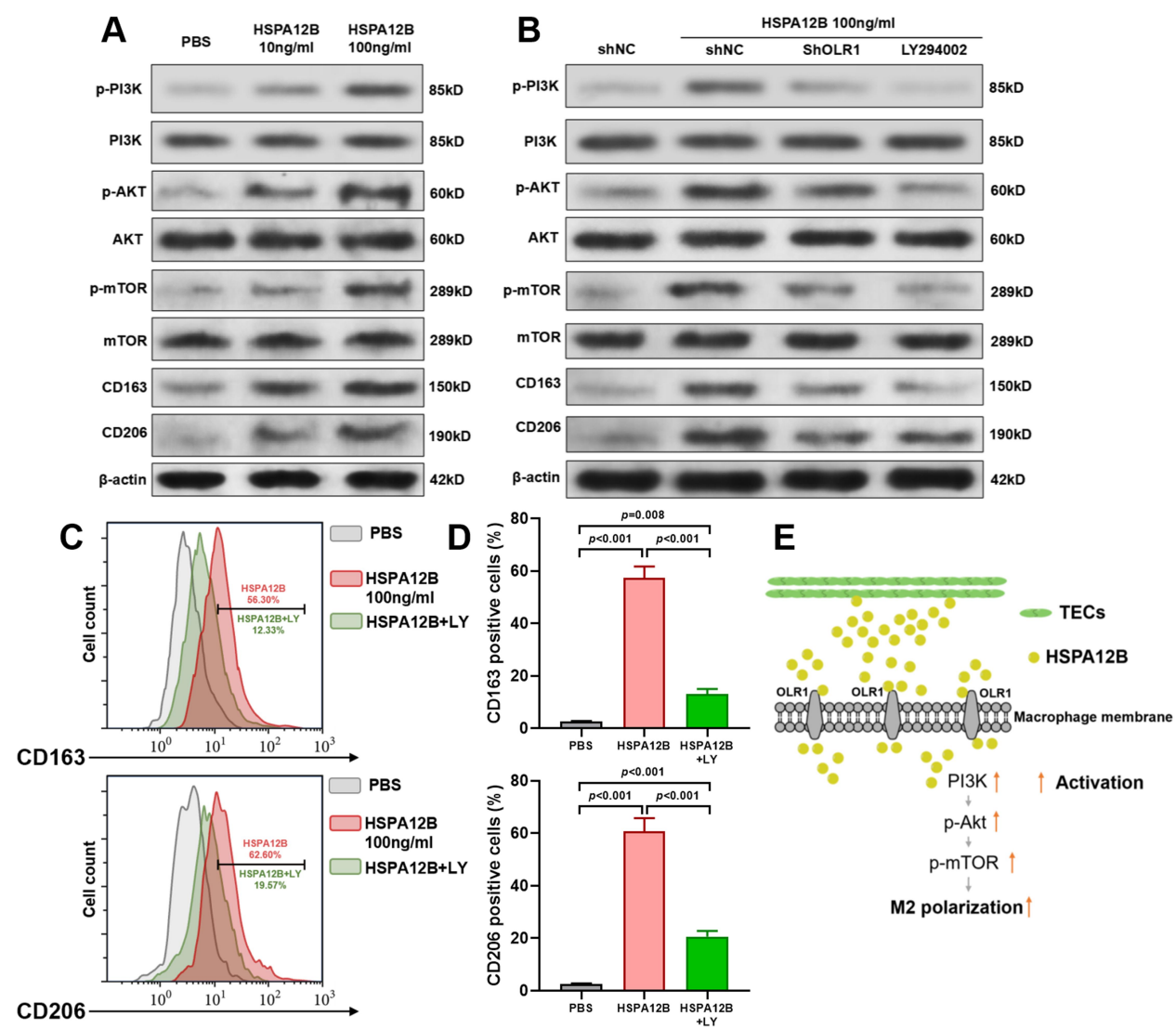

Figure 4 HSPAI2B activates the PI3K/Akt/mTOR signaling in macrophages. (A) Western blot assay of p-PI3K, PI3K, p-Akt, Akt, p-mTOR, mTOR, CDI63 and CD206 expression in RAW264.7 cells treated by $10 \mathrm{ng} / \mathrm{mL}$ or 100ng/mL HSPAI2B for 48h. (B) Western blot assay of p-PI3K, PI3K, p-Akt, Akt, P-mTOR, mTOR, CDI63 and CD206 expression in RAW264.7 cells treated by $100 \mathrm{ng} / \mathrm{mL}$ HSPAI2B alone or in combination with PI3K inhibitor LY294002 (LY, $10 \mu M$ ) and RAW264.7 with OLRI inhibition treated by $100 \mathrm{ng} / \mathrm{mL}$ HSPAI2B for 48h. (C and D) Representative images (C) and Statistical analysis (D) of markers for M2 macrophages (CDI63 and CD206) (D) in RAW264.7 cells 48h after treatment with $100 \mathrm{ng} / \mathrm{mL}$ HSPAI2B alone or in combination with PI3K inhibitor LY294002 (LY, $10 \mu M$ ). (E) A schematic image showing the speculative mechanism of TECs secreted HSPAI2B on M2 polarization of macrophages.

\section{Discussion}

Some recent studies revealed that HSPA12B might have important and complex roles in tumor biology. Transgenic mice overexpressing HSPA12B subjected to xenograft lung tumor models have significantly upregulated expression of VEGF and angiopoietin-1 and enhanced eNOS phosphorylation compared with wild-type counterparts. ${ }^{26}$ Its upregulation induces chemo-resistance in non-small-cell lung cancer ${ }^{24}$ and cervical squamous cell carcinoma. ${ }^{27}$ However, as a modulator of immune responses in endothelial cells, its functional role in tumor immune microenvironment has not yet been explored.

In the current study, we showed that PTECs had significantly elevated HSPA12B expression and secretion compared to normal HUVECs. Using bulk-seq data from over 500 primary HNSC cases, we observed significant positive correlations between HSPA12B expression and the expression of CD163 and CD206, two M2 macrophage markers. Functional assays based on flow cytometry analysis confirmed that exogenous HSPA12B treatment might stimulate the expression of M2 macrophage markers in a dose-dependent manner.

OLR1 is a human gene encodes oxidized low-density lipoprotein receptor 1 (OLR1), which is also known as lectin-type oxidized LDL receptor 1 (LOX-1). It is primarily expressed in endothelial cells, monocytes and smooth muscle cells ${ }^{28}$ and is the key receptor for oxidized low-density lipoproteins (ox-LDL) and HSP70 family proteins. ${ }^{29}$ Basal OLR1 expression is usually limited, but its expression is inducible via some proinflammatory and proatherogenic stimuli. ${ }^{30}$ Using sc-RNA-seq data, we demonstrated macrophage-associated OLR1 expression in HNSC tissues. Some recent studies reported that OLR1 expression was detected in some polymorphonuclear myeloid-derived suppressor cells (PMN-MDSC), which show potent immunesuppressive activity in cancer. ${ }^{31,32}$ It is also known that in the tumor microenvironment, high-ox-LDL can induce the upregulation of OLR1 in macrophages, which amplifies the 
high-ox-LDL signals and enhances M2 polarization. ${ }^{33}$ After internalization, Ox-LDL stimulates the generation of ROS, $\mathrm{NF}-\kappa \mathrm{B}$ activation, endoplasmic reticulum (ER) stress and subsequently M2 macrophage differentiation. ${ }^{34,35}$ These findings indicate a potential role of OLR1 in the immunesuppressive tumor microenvironment. Using sc-RNA-seq data in 98 macrophages, we observed positive correlations between OLR1 and CD163/CD206. In RAW264.7 cells, we demonstrated that OLR1 inhibition could significantly impair the uptake of exogenous HSPA12B and weaken HSPA12B induced M2 phenotypes. In addition, we showed that HSPA12B could activate the PI3K/Akt/mTOR signaling pathway in macrophages, the effect of which was mainly via OLR1. Based on these findings, we speculated that HSPA12B secreted by TECs might be taken by macrophages via OLR1 and induce M2 macrophage polarization via stimulating PI3K/Akt/mTOR signaling.

However, it is worth noticing that even with OLR1 knockdown, the entering of HSPA12B into RAW264.7 cells and HSPA12B induced CD163 and CD206 upregulation were not fully abrogated (Figure 3F-H) (Figure 3G and $\mathrm{H}$ ). Besides, blocking the activation of PI3K could not fully cancel the inducing effect of HSPA12B on M2 markers (Figure 4B-D). Therefore, we inferred that besides OLR1 mediated PI3K/Akt/mTOR signaling, there might be other receptors and/or channels of HSPA12B on the surface of macrophages and other signaling pathways involved in the activation of M2 polarization. Macrophages have considerable plasticity and thus can switch to different phenotypes upon different environmental stimuli. ${ }^{6}$ In some cases, macrophages may express both M1 and M2 markers during differentiation. ${ }^{6,36}$ Therefore, although we found that HSPA12 treatment did not influence the expression of CD86, a typically M1 marker in RAW264.7 cells, we could not exclude the possibility that HSPA12B might also induce M1 polarization. Future studies are required to explore more detailed signaling networking upon HSPA12B stimulation.

\section{Conclusion}

In summary, we speculated that aberrantly expressed and secreted HSPA12B by TECs could be taken by macrophages partly via OLR1, leading to subsequent activation of the $\mathrm{PI} 3 \mathrm{~K} / \mathrm{Akt} / \mathrm{mTOR}$ signaling pathway and elevated expression of M2 markers. This mechanism shows a novel cross-talk between TECs and TAMs, which contributes to the intratumoral immunosuppressive microenvironment.

\section{Funding}

No funding was received.

\section{Disclosure}

The authors report no conflicts of interest for this work.

\section{References}

1. Takahashi H, Sakakura K, Mito I, Ida S, Chikamatsu K. Dynamic changes in immune cell profile in head and neck squamous cell carcinoma: immunomodulatory effects of chemotherapy. Cancer Sci. 2016;107(8):1065-1071. doi:10.1111/cas. 12976

2. Chow LQM, Haddad R, Gupta S, et al. Antitumor activity of pembrolizumab in biomarker-unselected patients with recurrent and/or metastatic head and neck squamous cell carcinoma: results from the phase Ib KEYNOTE-012 expansion cohort. J Clin Oncol. 2016;34 (32):3838-3845. doi:10.1200/JCO.2016.68.1478

3. Larkins E, Blumenthal GM, Yuan W, et al. FDA approval summary: pembrolizumab for the treatment of recurrent or metastatic head and neck squamous cell carcinoma with disease progression on or after platinum-containing chemotherapy. Oncologist. 2017;22(7):873-878. doi:10.1634/theoncologist.2016-0496

4. O'Donnell JS, Long GV, Scolyer RA, Teng MW, Smyth MJ. Resistance to PD1/PDL1 checkpoint inhibition. Cancer Treat Rev. 2017;52:71-81. doi:10.1016/j.ctrv.2016.11.007

5. Zou W, Wolchok JD, Chen L. PD-L1 (B7-H1) and PD-1 pathway blockade for cancer therapy: mechanisms, response biomarkers, and combinations. Sci Transl Med. 2016;8(328):328rv324. doi:10.1126/ scitranslmed.aad7118

6. Evrard D, Szturz P, Tijeras-Raballand A, et al. Macrophages in the microenvironment of head and neck cancer: potential targets for cancer therapy. Oral Oncol. 2019;88:29-38. doi:10.1016/j. oraloncology.2018.10.040

7. Hao NB, Lu MH, Fan YH, Cao YL, Zhang ZR, Yang SM. Macrophages in tumor microenvironments and the progression of tumors. Clin Dev Immunol. 2012;2012:948098. doi:10.1155/2012/ 948098

8. Kumar AT, Knops A, Swendseid B, et al. Prognostic significance of tumor-associated macrophage content in head and neck squamous cell carcinoma: a meta-analysis. Front Oncol. 2019;9:656. doi: $10.3389 /$ fonc. 2019.00656

9. Edelman MJ, Wang X, Hodgson L, et al. Phase III randomized, placebo-controlled, double-blind trial of celecoxib in addition to standard chemotherapy for advanced non-small-cell lung cancer with cyclooxygenase-2 overexpression: CALGB 30801 (Alliance). $J$ Clin Oncol. 2017;35(19):2184-2192. doi:10.1200/JCO.2016. 71.3743

10. Butowski N, Colman H, De Groot JF, et al. Orally administered colony stimulating factor 1 receptor inhibitor PLX3397 in recurrent glioblastoma: an Ivy foundation early phase clinical trials consortium phase II study. Neuro Oncol. 2016;18(4):557-564. doi:10.1093/ neuonc/nov245

11. Reid T, Oronsky B, Scicinski J, et al. Safety and activity of RRx-001 in patients with advanced cancer: a first-in-human, open-label, dose-escalation Phase 1 study. Lancet Oncol. 2015;16 (9):1133-1142. doi:10.1016/S1470-2045(15)00089-3

12. Rosenzweig R, Nillegoda NB, Mayer MP, Bukau B. The Hsp70 chaperone network. Nat Rev Mol Cell Biol. 2019;20(11):665-680. doi:10.1038/s41580-019-0133-3

13. Steagall RJ, Rusinol AE, Truong QA, Han Z. HSPA12B is predominantly expressed in endothelial cells and required for angiogenesis. Arterioscler Thromb Vasc Biol. 2006;26(9):2012-2018. doi:10.1161/ 01.ATV.0000235720.61091.c7 
14. Wu J, Li X, Huang L, et al. HSPA12B inhibits lipopolysaccharide-induced inflammatory response in human umbilical vein endothelial cells. $J$ Cell Mol Med. 2015;19(3):544-554. doi:10.1111/jcmm.12464

15. Kong Q, Dai L, Wang Y, et al. HSPA12B attenuated acute myocardial ischemia/reperfusion injury via maintaining endothelial integrity in a pi3k/Akt/mTOR-dependent mechanism. Sci Rep. 2016;6(1):33636. doi:10.1038/srep33636

16. Zhao Y, Liu C, Liu J, et al. HSPA12B promotes functional recovery after ischaemic stroke through an eNOS-dependent mechanism. J Cell Mol Med. 2018;22(4):2252-2262. doi:10.1111/jcmm.13507

17. Puram SV, Tirosh I, Parikh AS, et al. Single-cell transcriptomic analysis of primary and metastatic tumor ecosystems in head and neck cancer. Cell. 2017;171(7):1611-1624. doi:10.1016/j. cell.2017.10.044

18. Feng Y, Liang Y, Zhu X, et al. The signaling protein Wnt5a promotes TGFbeta1-mediated macrophage polarization and kidney fibrosis by inducing the transcriptional regulators Yap/Taz. $J$ Biol Chem. 2018;293(50):19290-19302. doi:10.1074/jbc.RA118.005457

19. Li B, Sheng Z, Liu C, et al. Kallistatin inhibits atherosclerotic inflammation by regulating macrophage polarization. Hum Gene Ther. 2019;30(3):339-351. doi:10.1089/hum.2018.084

20. Radons J. The human HSP70 family of chaperones: where do we stand? Cell Stress Chaperones. 2016;21(3):379-404.

21. Wang X, Ding Z, Lin J, Guo Z, Mehta JL. LOX-1 in macrophage migration in response to ox-LDL and the involvement of calpains. Biochem Biophys Res Commun. 2015;467(1):135-139. doi:10.1016/j. bbrc.2015.09.100

22. Lara-Guzman OJ, Gil-Izquierdo A, Medina S, et al. Oxidized LDL triggers changes in oxidative stress and inflammatory biomarkers in human macrophages. Redox Biol. 2018;15:1-11. doi:10.1016/j. redox.2017.11.017

23. Ma Y, Lu C, Li C, et al. Overexpression of HSPA12B protects against cerebral ischemia/reperfusion injury via a PI3K/Akt-dependent mechanism. Biochim Biophys Acta. 2013;1832(1):57-66 doi:10.1016/j.bbadis.2012.10.003

24. Chen W, Liu X, Yuan S, Qiao T. HSPA12B overexpression induces cisplatin resistance in non-small-cell lung cancer by regulating the PI3K/Akt/NF-kappaB signaling pathway. Oncol Lett. 2018;15 (3):3883-3889. doi:10.3892/ol.2018.7800

25. Liu R, Fan T, Geng W, Chen YH, Ruan Q, Zhang C. Negative immune regulator TIPE2 promotes M2 macrophage differentiation through the activation of PI3K-AKT signaling pathway. PLoS One. 2017;12(1):e0170666. doi:10.1371/journal.pone.0170666
26. Ma H, Lu T, Zhang X, et al. HSPA12B: a novel facilitator of lung tumor growth. Oncotarget. 2015;6(12):9924-9936. doi:10.18632/ oncotarget. 3533

27. Yoshidomi K, Murakami A, Yakabe K, Sueoka K, Nawata S, Sugino N. Heat shock protein 70 is involved in malignant behaviors and chemosensitivities to cisplatin in cervical squamous cell carcinoma cells. J Obstet Gynaecol Res. 2014;40(5):1188-1196. doi:10. 1111/jog.12325

28. Draude G, Hrboticky N, Lorenz RL. The expression of the lectin-like oxidized low-density lipoprotein receptor (LOX-1) on human vascular smooth muscle cells and monocytes and its down-regulation by lovastatin. Biochem Pharmacol. 1999;57(4):383-386. doi:10.1016/ S0006-2952(98)00313-X

29. Murshid A, Theriault J, Gong J, Calderwood SK. Molecular chaperone receptors. Methods Mol Biol. 2018;1709:331-344.

30. Xu S, Ogura S, Chen J, Little PJ, Moss J, Liu P. LOX-1 in atherosclerosis: biological functions and pharmacological modifiers. Cell Mol Life Sci. 2013;70(16):2859-2872. doi:10.1007/s00018-0121194-z

31. Condamine T, Dominguez GA, Youn JI, et al. Lectin-type oxidized LDL receptor-1 distinguishes population of human polymorphonuclear myeloid-derived suppressor cells in cancer patients. Sci Immunol. 2016;1(2). doi:10.1126/sciimmunol.aaf8943.

32. Si Y, Merz SF, Jansen P, et al. Multidimensional imaging provides evidence for down-regulation of T cell effector function by MDSC in human cancer tissue. Sci Immunol. 2019;4(40). doi:10.1126/sciimmunol.aaw9159.

33. Seo JW, Yang EJ, Yoo KH, Choi IH. Macrophage differentiation from monocytes is influenced by the lipid oxidation degree of low density lipoprotein. Mediators Inflamm. 2015;2015:235797. doi:10.1155/2015/235797

34. Oh J, Riek AE, Weng S, et al. Endoplasmic reticulum stress controls M2 macrophage differentiation and foam cell formation. $J$ Biol Chem. 2012;287(15):11629-11641. doi:10.1074/jbc.M111.338673

35. Cominacini L, Pasini AF, Garbin U, et al. Oxidized low density lipoprotein (ox-LDL) binding to ox-LDL receptor-1 in endothelial cells induces the activation of NF-kappaB through an increased production of intracellular reactive oxygen species. J Biol Chem. 2000;275(17):12633-12638. doi:10.1074/jbc.275.17.12633

36. Guillot A, Tacke F. Liver macrophages: old dogmas and new insights. Hepatol Commun. 2019;3(6):730-743. doi:10.1002/hep4.1356
OncoTargets and Therapy

\section{Publish your work in this journal}

OncoTargets and Therapy is an international, peer-reviewed, open access journal focusing on the pathological basis of all cancers, potential targets for therapy and treatment protocols employed to improve the management of cancer patients. The journal also focuses on the impact of management programs and new therapeutic

Submit your manuscript here: https://www.dovepress.com/oncotargets-and-therapy-journa agents and protocols on patient perspectives such as quality of life, adherence and satisfaction. The manuscript management system is completely online and includes a very quick and fair peer-review system, which is all easy to use. Visit http://www.dovepress.com/ testimonials.php to read real quotes from published authors. 